\title{
Neumomediastino asociado a crisis asmática en el adulto. Reporte de dos casos
}

\author{
Andrés Fernando Rodríguez-Gutiérrez, *凶José A. Urrego, * Álvaro J. Burgos, *,* \\ Laura Navarro-Monterroza,* Danna Moros-Suárez,* Iván Moyano, Sol Naranjo-Casallas, \\ Nathalia Muñoz-Ortega*
}

* Universidad Nacional de Colombia. Bogotá, Colombia; ${ }^{\ddagger}$ Hospital de Engativá. Bogotá, Colombia.

RESUMEN. Introducción: El neumomediastino es la forma más frecuente del síndrome de fuga de aire en la crisis asmática del adulto. Es de curso benigno aunque puede complicarse por neumotórax y neumopericardio. Puede ser subdiagnosticado si no se sospecha. Presentación de los casos: Ambos casos se hallaron en Bogotá (2,640 metros sobre el nivel del mar) Caso 1: Mujer de 30 años con asma desde la infancia y tabaquismo ingresa por tos, disnea, dolor torácico anterior y cervical; se halló hipoxemia, broncoobstrucción y enfisema subcutáneo cervical, éste último confirmado en radiografía de tórax. La tomografía de tórax demostró neumomediastino extenso. Caso 2: Adolescente femenina de 16 años con antecedente de asma con síntomas respiratorios altos, disnea y dolor torácico; se documenta desaturación de oxígeno, enfisema subcutáneo cervical y torácico y sibilancias generalizadas. La radiografía de tórax confirmó enfisema subcutáneo con tomografía de tórax que diagnosticó neumomediastino. En ninguno de los casos se documentó neumotórax ni compromiso hemodinámico y ambos presentaron evolución satisfactoria con broncodilatadores, corticoide sistémico, oxigenoterapia y reposo. Conclusiones: El $11 \%$ de los pacientes con crisis asmática cursa con neumomediastino. La hipoxemia que no mejora a las 24 horas, el dolor torácico y cervical y el enfisema subcutáneo son pistas clínicas para sospecharlo siendo la tomografía de tórax el método diagnóstico por excelencia. El neumomediastino suele ser de curso benigno aunque podría complicarse poniendo en riesgo la vida. Su manejo consiste en el tratamiento de la broncoobstrucción, oxigenoterapia a alta fracción inspirada de $\mathrm{O} 2$ y reposo.

Palabras clave: Enfisema mediastínico, asma, enfisema subcutáneo, hipoxemia, tomografía [DeCS].

\section{Pneumomediastinum associated with asthmatic crisis in adulthood. A report of two cases}

ABSTRACT. Introduction: Pneumomediastinum (NM) is the most frequent manifestation of air leak syndrome in asthmatic crisis in adults. Its course is benign despite sometimes could occur complications like pneumothorax and pneumopericardium. However NM can be subdiagnosticated if it is not suspected. Cases presentation: Both cases was development in Bogota (2,640 m.a.s.I.) Case 1: A 30 years old female who had moderate persistent asthma since childhood and smoke habit presents with a history of cough, breathlessness, chest and cervical pain. On examination, she has hypoxemia, wheezing and cervical subcutaneous emphysema, the latter confirmed by chest $x$-ray. Chest computed tomography (CT) demonstrated extensive NM. Case 2: A 16 years old teenage women with a history of asthma since she was 8 years old was evaluated for acute respiratory symptoms, breathlessness and chest pain. On physical examination she has oxygen desaturation on room air, cervical and thoracic subcutaneous emphysema and generalized wheezing. Chest $x$-ray confirmed cervical subcutaneous emphysema and chest CT diagnosed NM. Neither of the cases had pneumothorax or hemodynamic compromise. Both cases had satisfactory evolution with bronchodilators, systemic corticoid, oxygen suplency an repose. Conclusions: Up to $11 \%$ of asthmatic crisis can course with NM. Clinical clues include no improvement of the hypoxemia in first 24 hours, chest and cervical pain and subcutaneous emphysema. Chest $\mathrm{CT}$ is the diagnostic tool of choice. NM usually follows a benign course although can has life-threatening complications. Management consists in treatment of bronchial obstruction, high fraction of inspired oxygen oxygenotherapie and repose.

Key words: Mediastinal emphysema, asthma, subcutaneous emphysema, hypoxemia, tomography [MeSH].

\section{$\triangle$ Autor para correspondencia:}

Dr. Andrés Fernando Rodríguez-Gutiérrez, Universidad Nacional

de Colombia. Bogotá, Colombia.

Correo electrónico: afrodriguezg@gmail.com

Trabajo recibido: 4-IX-2018; aceptado: 24-X-2018

\author{
Abreviaturas: \\ $\mathrm{CT}=$ Computed tomography \\ m.a.s.l. $=$ Meters above sea level \\ m.s.n.m. $=$ metros sobre el nivel del mar \\ $\mathrm{NM}=$ neumomediastino
}




\section{INTRODUCCIÓN}

La exacerbación del asma en el adulto constituye un amplio espectro de severidad, incluyendo crisis leves y de automanejo ambulatorio por el paciente hasta complicaciones severas que ponen en riesgo la vida, incluyendo la falla respiratoria, estado asmático e infección respiratoria baja. ${ }^{1}$ Aunque poco reconocido probablemente por subdiagnóstico, el síndrome de fuga de aire por localización anormal del mismo en múltiples ubicaciones es una complicación posible del asma exacerbado, y en una minoría de casos potencialmente mortal, siendo una entidad caracterizada principalmente en niños y con escasa literatura en el adulto. El neumomediastino (NM) es la forma más reconocida de síndrome de fuga de aire en asma, generalmente de evolución benigna. ${ }^{2,3}$ El objetivo del presente reporte es presentar dos casos de NM por crisis asmática en adultos, haciendo énfasis en sus características clínicas y diagnósticas.

\section{Caso 1}

Paciente femenina de 30 años, natural y procedente de Bogotá (2,640 metros sobre el nivel del mar [m.s.n.m]), trabajadora en labores domésticas y venta de juguetes, quien consultó al servicio de urgencias por cuadro de dos días de evolución que inició con odinofagia y tos seca de intensidad en aumento y posterior aparición de expectoración hialina que se asoció a disnea progresiva, síntomas que mejoraban con el uso de salbutamol. Adicionalmente y posterior a un episodio de acceso de tos fuerte, presentó aparición de dolor torácico de intensidad 10/10 en la escala numérica análoga, de localización anterior central irradiado a región pectoral izquierda y cara lateral del hemitórax izquierdo sin agravantes ni aliviantes. Negó fiebre, hemoptisis o cianosis. La paciente tenía antecedente de asma desde la infancia con múltiples crisis, última hacía un año; ninguna crisis había requerido hospitalización. Usaba salbutamol $200 \mu \mathrm{g}$ cada seis horas inhalado a necesidad y beclometasona $250 \mu \mathrm{g}$ cada 12 horas y refería tabaquismo hasta tres meses previos a la consulta a urgencias con un índice paquetes/año de 2 . Indicó que tenía un hijo que padecía asma.

El examen físico inicial reveló obesidad (índice de masa corporal de $33 \mathrm{~kg} / \mathrm{m}^{2}$ ), frecuencia cardíaca de 120 latidos por minuto, 28 respiraciones por minuto, tensión arterial de $128 / 74 \mathrm{mmHg}$, no tenía fiebre con pulso oximetría de pulso de $83 \%$ al ambiente. Tenía tirajes intercostales y supraclaviculares y se auscultaba disminución bilateral de los ruidos respiratorios con sibilancias espiratorias en ambos campos pulmo- nares y ruidos cardíacos taquicárdicos, sin hallazgos adicionales.

Fue valorada inicialmente por medicina general en urgencias que consideró crisis asmática, iniciando oxigenoterapia a 3 litros/minuto por cánula nasal, salbutamol y bromuro de ipratropio por micronebulizaciones, beclometasona $500 \mu \mathrm{g}$ inhalada cada 12 horas, metilprednisolona intravenosa (bolo intravenoso de $200 \mathrm{mg}$ y se continuó a $80 \mathrm{mg}$ intravenoso cada ocho horas) y sulfato de magnesio $2 \mathrm{~g}$ intravenoso en dosis única, así como cloruro de sodio a $0.9 \%$ a $60 \mathrm{ml} /$ hora.

Los gases arteriales realizados con fracción inspirada de oxígeno de 0.32 demostraron hipoxemia con falla terapéutica con trastorno moderado de la oxigenación y diferencia alvéolo arterial de oxígeno aumentada $\left(\mathrm{PaO}_{2}: 57.2 \mathrm{mmHg}, \mathrm{PA} / \mathrm{FI}: 179, \mathrm{D}(\mathrm{A}-\mathrm{a}) \mathrm{O}_{2}: 63.2 \mathrm{mmHg}\right)$ con hipoventilación alveolar $\left(\mathrm{PaCO}_{2}: 35 \mathrm{mmHg}\right.$, valores normales en Bogotá de $29 \mathrm{mmHg}$ a $33.5 \mathrm{mmHg})^{4}$ y acidemia mixta de predominio metabólico con Anion Gap normal (11.5 mEqu/l) asociado a hipercloremia de $108.6 \mathrm{mmol} / \mathrm{l}$. En el hemograma se observó hemoglobina de $15.8 \mathrm{~g} / \mathrm{dl}$, con plaquetas de 302.000 células/ $\mathrm{mcl}$, leucocitos de 14,170 células/mcl, neutrófilos de 13,260 células $/ \mathrm{mcl}$ y linfocitos de 580 células $/ \mathrm{mcl}$. La radiografía de tórax mostró hiperinsuflación y enfisema subcutáneo en cuello, sin signos que sugirieran ocupación alveolar (figura 1).

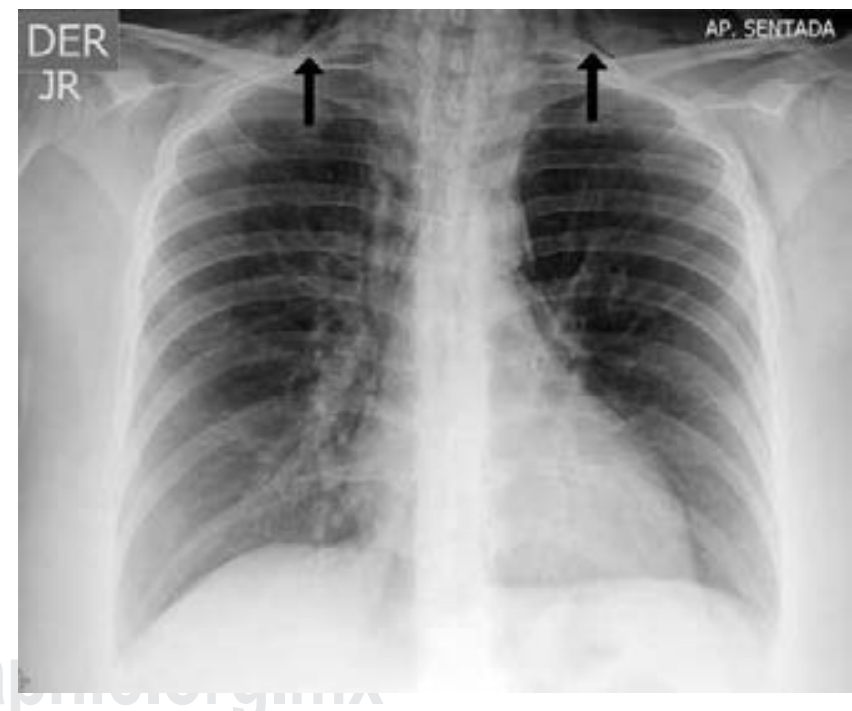

Fuente: Elaboración propia; tomada del caso.

Figura 1. Radiografía de tórax, proyecciones anteroposterior del caso 1. Sugiere signos de hiperinsuflación aérea dado por horizontalización de costillas, aplanamiento de los hemidiafragmas. Se observan en los tejidos blandos del cuello imágenes radiolúcidas irregulares que sugieren enfisema subcutáneo. 
La paciente fue valorada por el servicio de medicina interna; durante su estancia en observación del servicio de urgencias refiere aparición de dolor en región cervical anterior, haciéndose evidente al examen físico enfisema subcutáneo en ambos triángulos cervicales anteriores. Se sospecha neumomediastino asociado a barotrauma por crisis asmática severa. Se solicita tomografía de tórax que reveló neumomediastino extenso y enfisema subcutáneo (figura 2).

Se consideró NM espontáneo secundario a asma y decidió manejo conservador; se indicó reposo absoluto y medidas para evitar maniobras de Valsalva con metoclopramida $10 \mathrm{mg}$ intravenosa en caso de náuseas o emesis. Por persistencia del broncoespasmo se rotó a inhaloterapia con salbutamol $300 \mu \mathrm{g}$ cada cuatro horas, bromuro de ipratropio $40 \mu \mathrm{g}$ cada seis horas, se continuó corticoide sistémico con metilprednisolona a dosis de $40 \mathrm{mg}$ cada día con posterior paso a prednisolona $40 \mathrm{mg}$ por vía oral cada día, y analgesia con tramadol y acetaminofén. La paciente presentó mejoría progresiva del broncoespasmo y del enfisema subcutáneo, lográndose destete progresivo de la oxigenoterapia. Al quinto día de hospitalización, aún sin resolución de la broncoobstrucción, la paciente solicita salida voluntaria de la hospitalización por motivos personales, pese a las advertencias respecto a la necesidad de continuar vigilancia médica.

\section{Caso 2}

Paciente femenina de 16 años, con un cuadro de tres días de evolución que inició con prurito nasal, coriza, odinofagia, tos seca, fiebre y escalofríos. Tras dos días de estos síntomas inició con disnea de instauración progresiva y dolor torácico opresivo que al inicio se presentó a nivel de mitad superior de esternón y pos- teriormente se expandió hasta comprometer el tórax en todas sus caras, dolor de cuello y ambos hombros. El dolor se exacerbaba con la inspiración profunda e indicaba «sensación de burbujas en la piel». Reportó como antecedentes de importancia asma desde los ocho años e indicó varios episodios similares al actual que fueron manejados como crisis asmáticas.

Al examen físico presentaba taquipnea, uso de músculos accesorios de la respiración y una saturación arterial de oxígeno de $85 \%$ a ambiente. El resto de signos vitales eran normales. El tórax, cuello y espalda alta eran dolorosos y presentaban crépitos a la palpación, sugestivos de enfisema subcutáneo. La percusión torácica era normal. La auscultación pulmonar mostraba disminución global de los ruidos respiratorios y sibilancias espiratorias generalizadas.

Se realizó un electrocardiograma, que no presentaba hallazgos significativos. Asimismo, se solicitó una radiografía de tórax, donde se evidenció enfisema subcutáneo extenso (figura 3), por lo que se sospechó NM y se solicitó tomografía de tórax simple, que nuevamente evidenció un enfisema subcutáneo significativo pero, además, permitió confirmar presencia sustancial de NM (figura 4).

La paciente fue admitida a la unidad de cuidados intermedios para observación y manejo, donde se descartó un síndrome de Boerhaave. El tratamiento consistió en reposo, analgesia, oxígeno al 100\%, esteroides orales y broncodilatadores. La paciente egresó tras pocos días de hospitalización con notoria mejoría en sus signos y síntomas.

\section{DISCUSIÓN}

Se presentan dos casos de NM probablemente secundarios a crisis asmática. El término NM fue

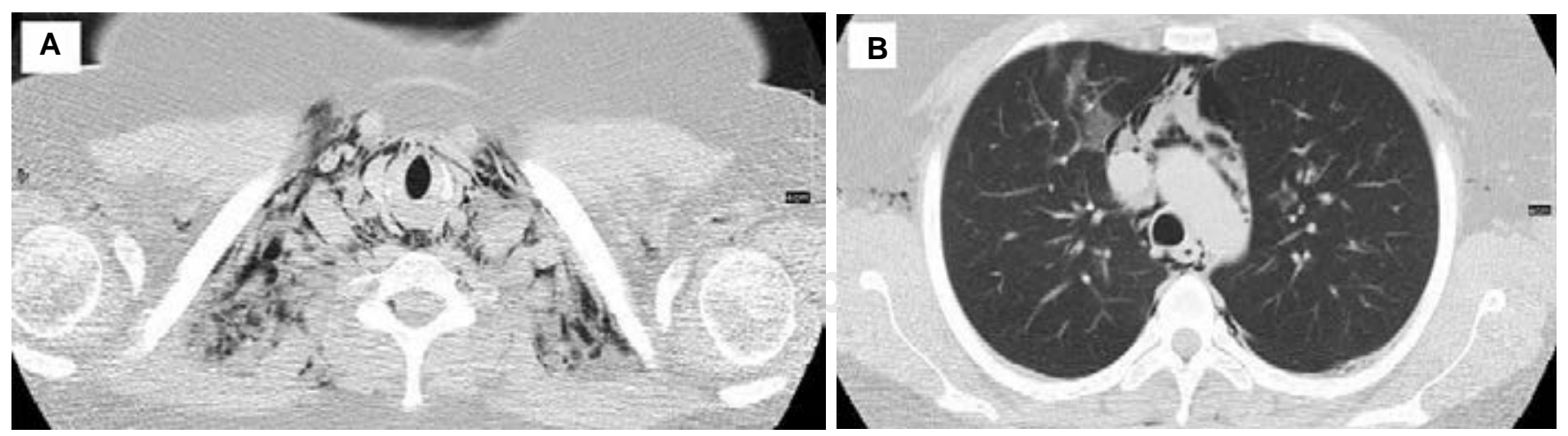

Fuente: Elaboración propia; tomada del caso.

Figura 2. Tomografía de tórax simple, ventana para pulmón en cortes axiales. Se observa en A: enfisema en los tejidos blandos del cuello supravalvular (también tenía en región axilar y de la pared anterior de tórax) secundario en B: neumomediastino extenso. 


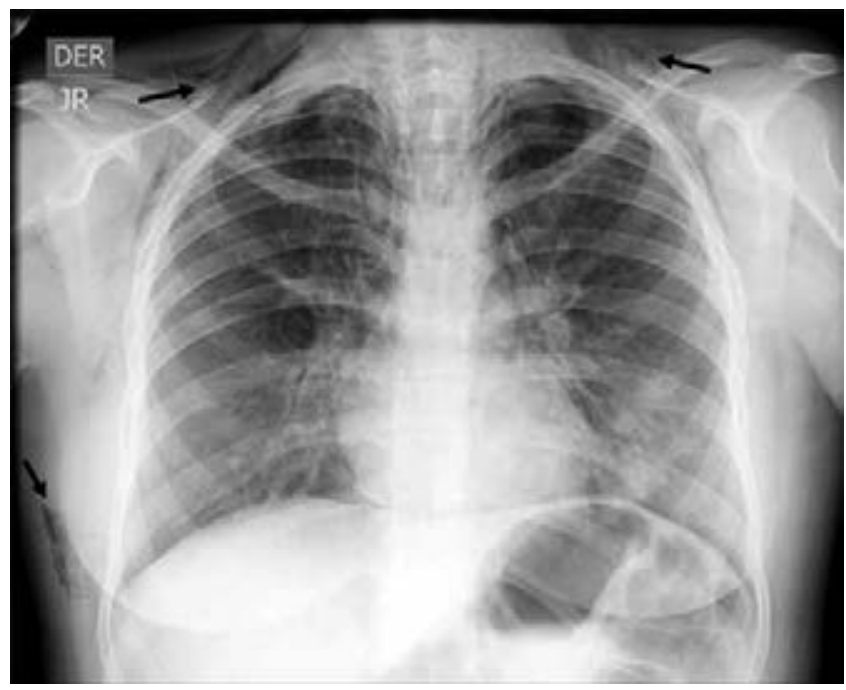

Fuente: Elaboración propia; tomada del caso.

Figura 3. Radiografía de tórax anteroposterior del caso 2. Muestra enfisema subcutáneo (flechas).

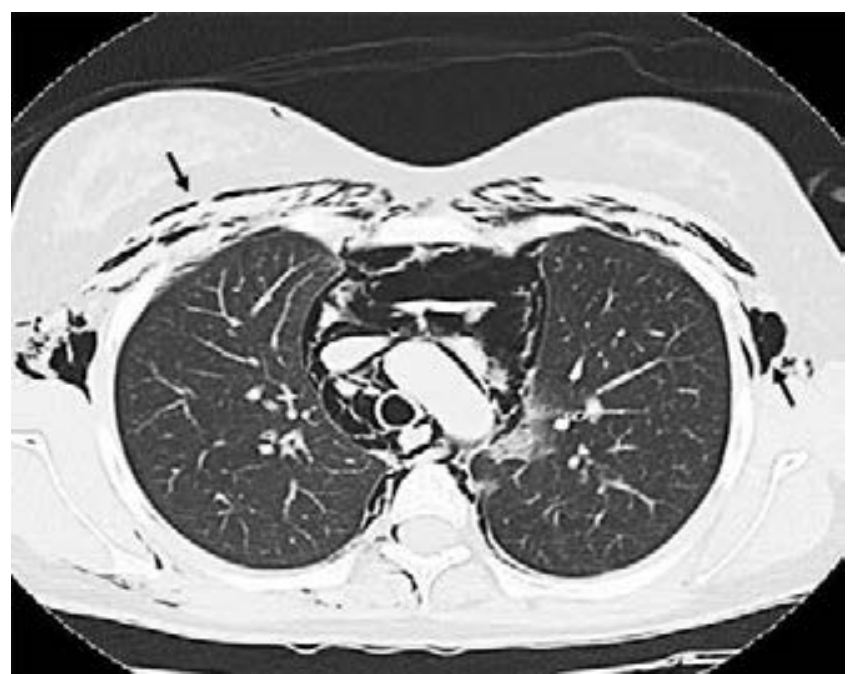

Fuente: Elaboración propia; tomada del caso.

Figura 4. Tomografía de tórax simple en corte axial del caso 2 que muestra abundante aire mediastinal a nivel del arco aórtico. Puede también verse enfisema subcutáneo en cada cara del tórax (flechas).

introducido por primera vez a la literatura médica por Hamman en $1939,{ }^{5}$ aunque las primeras descripciones conocidas de la entidad corresponden a Laennec a principios del siglo XIX. ${ }^{6}$ Su clasificación no es del todo clara en la literatura, ${ }^{7,8}$ aunque la diferenciación entre traumático VS. no traumático y este último entre espontáneo (sin causa establecida)
VS. secundario (e.g. por asma) parece ser la forma más razonable.

Como lo demuestran los casos aquí presentados, el NM no es la única complicación asociada a la localización anormal de aire proveniente del árbol bronquial; también se incluye el enfisema subcutáneo en cara, cuello y región superior del tórax, el neumotórax, neumopericardio y la eventual extensión hasta el espacio epidural (neumorraquis). ${ }^{9}$ El crisol clínico sindromático derivado de dicha localización anormal de aire se ha denominado "síndrome de fuga de aire». ${ }^{2}$

El proceso patofisiológico de la migración del aire suele tener como evento iniciador la ruptura de la continuidad de los alvéolos marginales, que son aquellos periféricos o que tienen contacto con estructuras diferentes a otros alvéolos y por tanto menor capacidad de descargar el aire que les ingresa a través de los poros de Kohn. El alvéolo roto genera inicialmente enfisema intersticial pulmonar, con extensión centrípeta del aire a través de la vaina broncovascular hasta el hilio pulmonar donde finalmente alcanza el mediastino. El aumento de presión en el mediastino puede condicionar el paso del aire a los tejidos conectivos laxos del cuello, el tórax, el pericardio o el espacio epidural. Dicho proceso patofisiológico fue propuesto por los doctores Macklin en 1939 y 1944 por lo que es conocido como «efecto Macklin».10,11

El efecto Macklin es el efecto final de múltiples etiologías del NM, incluyendo el trauma cerrado de tórax, la ventilación mecánica por presión positiva o el asma; en este último caso las probables formas de ruptura de los alvéolos marginales son el volutrauma y el barotrauma asociado a la hiperinsuflación dinámica y las maniobras de Valsalva por tos. Un mecanismo alternativo al efecto Macklin de origen de la fuga de aire hacia el mediastino es la ruptura espontánea del esófago asociado a episodios de emesis, clínicamente configurando el síndrome de Boerhaave, entidad de alta morbimortalidad y diagnóstico diferencial a considerar. ${ }^{12}$

La incidencia del NM durante los episodios de agudización del asma no es clara, aunque en el adulto podría ser relativamente alta. En una cohorte de pacientes adultos con crisis asmática en un hospital de Italia se realizó tomografía de tórax a quienes no mejoraron la oxigenación a las 24 horas de inicio del tratamiento; dicha estrategia demostró una incidencia del $11 \%$ de NM entre todos los pacientes con la crisis. ${ }^{3}$ En una cohorte de pacientes pediátricos en los años setenta, sin acceso a tomografía de tórax y usando la clínica más la radiografía de tórax como método diagnóstico, se encontró una incidencia de síndrome de fuga de aire de uno por cada mil casos de exacerbación; la mitad de los casos encontrados fueron NM. ${ }^{2}$ Otra cohorte 
de pacientes pediátricos en los años noventa, sin uso de tomografía, reportó una incidencia de $0.3 \% \cdot{ }^{13}$ Esta marcada discrepancia de la incidencia de NM entre adultos y niños en crisis asmática indica que es probable la existencia de diferencias patofisiológicas; esta hipótesis está apoyada por el estudio de Eggleston et al., quienes demostraron que en niños la incidencia de NM aumenta notoriamente con la edad. ${ }^{14}$ Sin embargo, la diferencia también se explica en que el estudio en adultos presentado usó tomografía de tórax, ${ }^{3}$ que mejora notoriamente la capacidad de diagnóstico.

Como posibles factores de riesgo del NM por asma se ha establecido edad joven (tercera década de la vida) y sensibilización a Alternaria spp. ${ }^{3}$ Aunque no es claro en NM por asma, en el caso del NM espontáneo se ha asociado al tabaquismo como condición predisponente entre un 21 y $34 \%{ }^{15,16}$ Esto es notorio ya que la paciente presentada en el caso 1 era fumadora, teniendo en cuenta que lo había suspendido hacía tres meses.

En los casos presentados se hizo evidente en la presentación clínica la presencia de tos, hipoxemia, enfisema subcutáneo, dolor torácico y en el caso 1 dolor cervical. En su presentación clínica el NM por asma suele implicar menores niveles de saturación de oxihemoglobina, lo cual puede ser usado como una guía clínica para sospecharlo., ${ }^{3,13}$ El NM por asma probablemente tiene comportamiento sindromático similar del NM espontáneo, que incluye principalmente el dolor torácico y el enfisema subcutáneo. El dolor torácico suele ser retroesternal irradiado a cuello. Sahni et al. realizaron una revisión de seis series de casos reuniendo 201 pacientes con NM espontáneo y encontraron que los síntomas más frecuentes eran el dolor torácico (75\%), la disnea (49\%), el dolor en cuello (39\%) y la tos (36\%). Otros síntomas menos frecuentes incluyen disfagia, odinofagia, debilidad, disfonía y dolor dorsal. En cuanto al examen físico, los hallazgos encontrados con más frecuencia en el mismo estudio fueron el enfisema subcutáneo y el signo de Hamman, en el 58 y $18 \%$ de los casos, respectivamente. ${ }^{8}$ Este último consiste en la auscultación de crépitos sobre el mediastino sincrónicos con los latidos cardíacos, signo que si bien se acuñó más recientemente, su descripción ya se encontraba en los textos de Laennec del siglo XIX. ${ }^{6,17}$

El diagnóstico de NM tiene como primera línea diagnóstica a la radiografía de tórax. Varios signos radiológicos han sido descritos (signo de Spinnaker, signo del anillo alrededor de la arteria, signo del diafragma continuo, entre otros), que en general dependen de la delimitación de las estructuras mediastinales y perimediastinales por los depósitos de aire en sus contornos. ${ }^{18-20}$ Sin embargo, dado que la radiografía de tórax puede ser normal en parte sustancial de los pacientes con exacerbación por asma ${ }^{3}$ y teniendo en cuenta que el diagnóstico es frecuentemente pasado por alto, ${ }^{21}$ la tomografía de tórax debería ser usada en algunos casos para confirmar la presencia de NM. ${ }^{20}$

En ausencia de estudios de calidad que validen determinados manejos, el tratamiento del NM por asma se extrapola del NM espontáneo, que a su vez se fundamenta actualmente en la experiencia clínica, series de casos, extrapolaciones de otras entidades y en el excelente pronóstico que generalmente acarrea, dado que en casi la totalidad de los casos el aire se reabsorberá en 2-15 días. , 22,23 De este modo, el manejo es conservador con analgesia y reposo para evitar maniobras de Valsalva, manejo de la causa de base y el oxígeno a alto flujo. ${ }^{23,24}$ Esto último facilita la reabsorción del aire al reemplazar las moléculas de nitrógeno en alvéolos, tejido conectivo y mediastino por moléculas de oxígeno de más fácil y rápida absorción. ${ }^{25,26}$ No parece ser necesaria la restricción a la alimentación. ${ }^{27}$ Basados en una escasa literatura de baja calidad existente al respecto, en los casos donde se produzca un NM a tensión, una mediastinotomía podría resultar de utilidad. ${ }^{28}$ Asimismo, la improbable presencia de taponamiento cardíaco por neumopericardio requeriría el drenaje de este aire, que en otros contextos ha mostrado mejoría hemodinámica dramática. ${ }^{29}$

El curso del NM en asma parece ser benigno y autolimitado, sin aumentar la mortalidad, la necesidad de intervenciones adicionales ni extensión de la estancia hospitalaria. ${ }^{3}$ Sin embargo, debe tenerse en cuenta que hasta un $20 \%$ de los casos de asma fatal se ha reportado con enfisema intersticial en series de autopsias. ${ }^{30,31}$ Asimismo, el riesgo de recurrencia es bajo; en el escenario del NM espontáneo es menor del $2 \%$ en las series de casos. ${ }^{27}$

\section{CONCLUSIONES}

El síndrome de fuga de aire es una complicación a tener en cuenta en las crisis asmáticas. El NM es la forma más frecuente del mismo, estando presente hasta en uno de cada diez pacientes adultos que consultan a urgencias por crisis asmática. El dolor torácico, el enfisema subcutáneo y la no mejoría de la hipoxemia pese al manejo adecuado de la crisis son elementos orientadores en la sospecha del NM. La radiografía de tórax es útil si se encuentran hallazgos, pero su negatividad no excluye el diagnóstico; si la sospecha clínica de NM persiste, está indicada la toma de tomografía de tórax que tiene alta sensibilidad para la detección de dicha entidad. El $\mathrm{NM}$ en asma es en general de curso benigno y en la mayoría de los casos requerirá reposo, oxigenoterapia con alta fracción inspirada de oxígeno y el manejo de 
base de la crisis. Eventualmente podría progresar a neumopericardio, neumotórax, neumorraquis e hipertensión mediastinal, aunque son complicaciones infrecuentes. En este caso la descompresión por mediastinotomía con tubo puede ser necesaria. La literatura y estudios del NM por asma en el adulto son escasos, es necesario profundizar la investigación de esta entidad.

\section{Declaración de transparencia}

Los autores declaran que los casos clínicos presentados son expuestos de forma completa y honesta, que no se han omitido detalles esenciales del curso del mismo y que se han informado todas las limitaciones durante su desarrollo.

\section{Financiación}

No se recibió ningún tipo de financiación.

\section{Conflicto de intereses}

Los autores no declaran.

\section{Agradecimientos}

En especial al equipo asistencial del Hospital de Engativá.

\section{REFERENCIAS}

1. Ichinose $M$, Sugiura $H$, Nagase $H$, et al. Japanese guidelines for adult asthma 2017. Allergol Int 2017;66(2):163-189. doi: 10.1016/j.alit.2016.12.005

2. Takeishi T, Nishima S, Kano S. Air leak syndrome (ALS) as complication of asthma. Acta Paediatr Jpn 1989;31(3):330-334. doi: 10.1111/j.1442-200X.1989. tb01309.x.

3. Vianello A, Caminati M, Chieco-Bianchi F, et al. Spontaneous pneumomediastinum complicating severe acute asthma exacerbation in adult patients. J Asthma 2017:1-7. doi: 10.1080/02770903.2017.1388392.

4. Maldonado D, González-García M, Barrero M, Casas A, Torres-Duque CA. Reference values for arterial blood gases at an altitude of 2.640 meters. Am J Respir Crit Care Med 2013;187:A4852. https://goo.gl/bdMjqu

5. Hamman L. Spontaneous mediastinal emphysema. Bull Johns Hopkins Hosp 1939;64:1-21

6. Laennec RTH. A treatise on diseases of the chest and on mediate auscultation, translated by Jhon Forbes. 2 ed. Underwood, London: T \& G; 1827. https://goo.gl/ DX1Ww4

7. Kouritas VK, Papagiannopoulos K, Lazaridis G, et al. Pneumomediastinum. J Thorac Dis 2015;7(Suppl 1):S44-S49. doi: 10.3978/j.issn.2072-1439.2015.01.11
8. Sahni S, Verma S, Grullon J, Esquire A, Patel P, Talwar A. Spontaneous pneumomediastinum: time for consensus. N Am J Med Sci 2013;5(8):460-464. doi: 10.4103/19472714.117296.

9. Liu YW, Kao CN, Wang YM. Pneumorrhachis: an under-recognized entity correlates with severity of spontaneous pneumomediastinum. J Thorac Dis 2018;10(2):E149-E151. http://jtd.amegroups.com/article/ view/18658

10. Macklin CC. Transport of air along sheaths of pulmonic blood vessels from alveoli to mediastinum: Clinical implications. Arch Intern Med 1939;64(5):913-926. doi: 10.1001/archinte.1939.00190050019003. doi: 10.1001/ archinte.1939.00190050019003.

11. Macklin MT, Macklin CC. Malignant interstitial emphysema of the lungs and mediastinum as an important occult complication in many respiratory diseases and other conditions: interpretation of the clinical literature in the light of laboratory experiment. Medicine 1944;23:281-358. https://goo.gl/GRT2ph

12. Glatz T, Marjanovic G, Kulemann B, et al. Management and outcome of esophageal stenting for spontaneous esophageal perforations. Dis Esophagus 2017;30(3): 1-6. doi: 10.1111/dote.12461.

13. Stack AM, Caputo GL. Pneumomediastinum in childhood asthma. Pediatr Emerg Care 1996;12(2):98-101. https:// goo.gl/3yfkXR

14. Eggleston PA, Ward BH, Pierson WE, Bierman CW. Radiographic abnormalities in acute asthma in children. Pediatrics 1974;54(4):442-449. https://goo.gl/mebk4H

15. Macia I, Moya J, Ramos R, et al. Spontaneous pneumomediastinum: 41 cases. Eur J Cardiothorac Surg 2007;31(6):1110-1114. doi: 10.1016/j.ejcts.2007.03.008.

16. Park SJ, Park JY, Jung J, Park SY. Clinical Mmnifestations of Spontaneous Pneumomediastinum. Korean J Thorac Cardiovasc Surg 2016;49(4):287-291. doi: 10.5090/ kjtcs.2016.49.4.287.

17. Bodey GP. Medical mediastinal emphysema. Ann Intern Med 1961;54:46-56. doi: 10.7326/0003-4819-54-1-46.

18. Bejvan SM, Godwin JD. Pneumomediastinum: old signs and new signs. AJR Am J Roentgenol 1996;166(5):10411048. doi: 10.2214/ajr.166.5.8615238.

19. Lawal TA, Glüer S, Reismann M, Dördelmann M, Schirg E, Ure B. Spontaneous neonatal pneumomediastinum: the "spinnaker sail" sign. Eur J Pediatr Surg 2009;19(1):5052. doi: $10.1055 / \mathrm{s}-2008-1038393$.

20. Zylak CM, Standen JR, Barnes GR, Zylak CJ. Pneumomediastinum revisited. Radiographics 2000;20(4):1043-1057. doi: 10.1148/ radiographics.20.4.g00jl131043.

21. Yellin A, Gapany-Gapanavicius M, Lieberman Y. Spontaneous pneumomediastinum: is it a rare cause of chest pain? Thorax 1983;38(5):383-385. doi: 10.1136/ thx.38.5.383.

22. Dekel B, Paret G, Szeinberg A, Vardi A, Barzilay Z. Spontaneous pneumomediastinum in children: clinical and natural history. Eur J Pediatr 1996;155(8):695-697. https://goo.gl/3B9vzZ 
23. Gerazounis M, Athanassiadi K, Kalantzi N, Moustardas M. Spontaneous pneumomediastinum: a rare benign entity. J Thorac Cardiovasc Surg 2003;126(3):774-776. doi: 10.1016/S0022-5223(03)00124-7.

24. Fitzwater JW, Silva NN, Knight CG, Malvezzi L, Ramos-Irizarry C, Burnweit CA. Management of spontaneous pneumomediastinum in children. J Pediatr Surg 2015;50(6):983-986. doi: 10.1016/j. jpedsurg.2015.03.024.

25. Munsell WP. Pneumomediastinum. A report of 28 cases and review of the literature. JAMA 1967;202(8):689-693. doi: 10.1001/jama.1967.03130210063009.

26. Patel A, Kesler B, Wise RA. Persistent pneumomediastinum in interstitial fibrosis associated with rheumatoid arthritis: treatment with high-concentration oxygen. Chest 2000;117(6):1809-1813. doi: 10.1378/ chest.117.6.1809.

27. Kim KS, Jeon HW, Moon Y, et al. Clinical experience of spontaneous pneumomediastinum: diagnosis and treatment. J Thorac Dis 2015;7(10):1817-1824. doi: 10.3978/j.issn.2072-1439.2015.10.58.

28. Herlan DB, Landreneau RJ, Ferson PF. Massive spontaneous subcutaneous emphysema. Acute management with infraclavicular "blow holes". Chest 1992;102(2):503-505. doi: doi.org/10.1378/ chest.102.2.503.

29. Reddy PS, Curtiss EI, O'Toole JD, Shaver JA. Cardiac tamponade: hemodynamic observations in man. Circulation 1978;58(2):265-672. https://goo.gl/DZtjHh

30. Cluroe A, Holloway L, Thomson K, Purdie G, Beasley R. Bronchial gland duct ectasia in fatal bronchial asthma: association with interstitial emphysema. J Clin Pathol 1989;42(10):1026-1031. https://goo.gl/op3wPS

31. Mauad T, Nascimento FBPD, Dolhnikoff M, Picka MCM, Saldiva PHN; BIAS. Pulmonary interstitial emphysema in fatal asthma: case report and histopathological review. BMC Pulm Med 2018;18(1):50. doi: 10.1186/s12890-0180615-7. 